\title{
Mucinous carcinoma of the colon: correlation of loss of mismatch repair enzymes with clinicopathologic features and survival
}

\author{
Sanjay Kakar ${ }^{1}$, Saime Aksoy², Lawrence J Burgart ${ }^{2}$ and Thomas C Smyrk² \\ ${ }^{1}$ Department of Pathology, University of California San Francisco and Veteran Affairs Medical Center, San \\ Francisco, CA, USA and ${ }^{2}$ Mayo Clinic, Rochester, MN, USA
}

\begin{abstract}
Colorectal carcinoma with microsatellite instability (MSI-H) has a characteristic clinicopathologic profile, typically forming right-sided, lymphocyte-rich tumors that are often mucinous. Mucinous histology in general has been linked to adverse prognosis in some studies, but not in others. MSI-H carcinoma, in contrast, has a better prognosis than microsatellite stable carcinoma in most studies. We assessed the relationship between MSI status, clinicopathologic features and outcome for 248 consecutive patients with resected mucinous carcinoma. All cases were reviewed to confirm mucinous histology. Immunohistochemical stains for DNA mismatch repair enzymes hMLH1, hMSH2 and hMSH6 were performed on a representative block from each case. Tumors lacking expression of a mismatch repair enzyme were designated MSI-H; all others were classified as microsatellite stable. Age, sex, tumor size, site, grade, stage, growth pattern, Crohn's-like reaction, vascular invasion and number of tumor-infiltrating lymphocytes were evaluated without knowledge of MSI status or patient outcome. $72(29.3 \%)$ mucinous carcinomas were MSI-H. Compared to microsatellite stable mucinous cancers, they were more likely to be right-sided (83.3 vs $48.6 \%, P<0.001)$, have a Crohn's -like reaction (65.7 vs $29.8 \%, P<0.001)$ and have many tumor infiltrating lymphocytes $(72.2$ vs $20.8 \%, P<0.001)$. MSI-H mucinous cancers presented more often as localized disease (66.7 vs $38.1 \%, P<0.001)$ and less often with lymph node (26.4 vs $44.9 \%$ ) or distant (4.2 vs $16.5 \%$ ) metastases. In univariate analysis, MSI had a favorable effect on ageadjusted survival (hazard ratio $0.597, P=0.02$ ). In multivariate analysis, age, grade, Crohn's-like reaction and stage were independent predictors of survival, but MSI status was not. In conclusion, MSI-H mucinous carcinomas are right-sided, low-stage tumors with Crohn's-like reaction and tumor-infiltrating lymphocytes. The outcome for MSI-H mucinous carcinoma is better than that of microsatellite-stable mucinous carcinoma, but MSI status is not an independent predictor of survival.
\end{abstract}

Modern Pathology (2004) 17, 696-700, advance online publication, 12 March 2004; doi:10.1038/modpathol.3800093

Keywords: mucinous carcinoma; colon; rectum; MSI; mismatch repair; survival

All colorectal carcinomas arising in the setting of Hereditary Nonpolyposis Colorectal Cancer (HNPCC) demonstrate high-level microsatellite instability (MSI-H), as do $15-20 \%$ of sporadic colorectal cancers. ${ }^{1-3}$ The phenomenon reflects accumulated errors in DNA microsatellite repeat sequences following inactivation of a DNA mismatch repair enzyme via mutation (in the hereditary setting) or hypermethylation of the promoter region (in sporadic cancers). ${ }^{2,3}$ Whether hereditary or sporadic, MSI$\mathrm{H}$ tumors characteristically involve the right side of the colon and show medullary, mucinous or signet

Correspondence: Dr TC Smyrk, MD, Department of PathologyMayo Clinic, 200 First St SW, Rochester, MN 55905, USA.

E-mail: smyrk.thomas@mayo.edu

Received 5 November 2003; revised 20 January 2004; accepted 21 January 2004; published online 12 March 2004 ring histology. ${ }^{4-7}$ Host lymphoid response in the form of tumor infiltrating lymphocytes or peritumoral Crohn's-like infiltrate is usually prominent. ${ }^{7,8}$ In most studies, patients with MSI-H colorectal cancer have better prognosis than do those with microsatellite stable carcinoma. ${ }^{9-16}$

Mucinous carcinoma is a histologic subtype constituting $11-15 \%$ of colorectal cancer. ${ }^{17-21}$ Mucinous histology has been shown to be an independent adverse prognostic factor in some studies, ${ }^{19,21-23}$ but not in others. ${ }^{17,18,20,24,25}$ The current consensus of the College of American Pathologists (CAP) and American Joint Committee on Cancer is that mucinous differentiation is not proven as a statistically significant prognostic factor independent of histologic grade. ${ }^{26,27}$ The CAP has recommended that tumor type (like mucinous carcinoma) should be correlated with outcome after adjustment for MSI 
status in statistically robust studies with multivariate analysis, in order to definitively determine its prognostic significance'. ${ }^{27}$ We studied associations between MSI status, clinicopathologic features and survival in a consecutive series of 248 resected mucinous carcinomas.

\section{Methods}

The study population comprised 248 patients who had a mucinous carcinoma of the colorectum resected during the years 1984-2001 at the Mayo Clinic. Age, gender, tumor size and site were obtained from the pathology report. Tumors in the cecum, ascending colon and transverse colon were classified as right-sided and those in the descending colon, sigmoid and rectum were left-sided. Clinical charts were reviewed to obtain information about adjuvant therapy and the presence of underlying conditions such as inflammatory bowel disease, familial adenomatous polyposis and hereditary nonpolyposis colorectal cancer. Survival data were obtained from the Mayo tumor registry; clinical follow-up ranged from 1 month to 16 years (mean 5.8 years). The study was approved by the Institutional Review Board.

Histologic parameters were evaluated on hematoxylin-eosin-stained slides without knowledge of MSI status. Two to five slides were examined in each case (average 3). By definition, all tumors had mucin constituting more than $50 \%$ of tumor volume. The presence of additional patterns (medullary, signet ring cell) was noted. Tumors were grades 1-4 depending on the degree of gland formation: $>75 \%$, grade $1,50-75 \%$, grade $2,25-50 \%$, grade 3 , and $<25 \%$, grade 4 . The growth pattern was recorded as pushing when the advancing front of the tumor was sharply defined, and infiltrating if there was irregular infiltration of tumor cells at the tumor-stroma interface. Tumor-infiltrating lymphocytes (TIL) were counted in 10 high-power fields and expressed as average count per high-power field. Crohn's-like reaction was recorded as positive or negative; it was regarded as positive only when there were two or more lymphoid aggregates per glass slide. The presence of vascular invasion was noted. Depth of tumor invasion and lymph node status were assessed and used in conjunction with clinical information to determine tumor stage according to the Astler-Coller modification of Dukes' staging.

Mouse monoclonal antibodies against hMLH1, hMSH2 and hMSH6 were used for immunohistochemistry. Formalin-fixed paraffin-embedded sections were rehydrated using xylene and graded alcohol washes. After blocking the endogenous peroxidase, heat-induced antigen retrieval was performed with EDTA buffer, pH 8.0 in a steamer for $5 \mathrm{~min}$, and the sections were incubated with primary antibody (hMLH1 1:200, Pharmingen,
hMSH2 1:50, Oncogene Sciences and hMSH6 1:500, Transduction Laboratories) for $60 \mathrm{~min}$. Following washing with PBS, sections were incubated with the biotinylated goat anti-mouse secondary antibody (1:100) for $30 \mathrm{~min}$. Sections were incubated with avidin-biotin complex (ABC elite kit, Vector laboratories, Burlingame, CA, USA) for $20 \mathrm{~min}$, developed with diacetyl benzidine (DAB) for 5$10 \mathrm{~min}$ and counterstained with hematoxylin. The adjacent normal colonic mucosa and stromal cells with intact mismatch repair function served as the internal control. Immunohistochemistry results were classified as positive or negative. Any nuclear staining was considered a positive result. Tumors with no nuclear staining for hMLH1, hMLH2 and/or hMLH6 were classified as MSI-H. All other cases were regarded as microsatellite stable (MSS).

The relationship between MSI and various clinicopathologic features was tested by $\chi^{2}$-test for discrete variables, Mantel-Hanzel test for categorical variables and rank sum test for continuous variables. In the survival analysis, the starting point for the survival time was the date of surgery. Survival curves were calculated using the KaplanMeier method and statistical significance between curves was tested by the log-rank test. Univariate analysis was performed using multivariate Cox proportional hazards model; the hazard ratio and its 95\% confidence interval (CI) were assessed for each factor. $P$-values of $<0.05$ were considered statistically significant.

\section{Results}

In all, $72(29.3 \%)$ mucinous carcinomas were designated MSI-H on the basis of loss of staining for hMLH1 $(n=69)$ or hMSH2 $(n=3)$. Staining for hMSH6 was lost in all the three cases lacking hMLH2, but not in any others. Four mucinous carcinomas arose in the setting of inflammatory bowel disease. None of the 248 patients carried a diagnosis of a hereditary syndrome as judged by the review of patient charts.

MSI-H cancers were more likely to be right-sided, low-stage tumors with Crohn's-like reaction, numerous TILs and mixed histologic patterns (Table 1). There was no correlation between MSI status and sex, tumor size, grade, growth pattern or vascular invasion. MSI-H tumors tended to occur at an older age, but the correlation was not statistically significant. There was no significant difference in the number of patients receiving adjuvant therapy for MSI-H cancer vs MSS cancer: $22.2 \%$ of MSI-H stage $\mathrm{B}$ cancers received adjuvant therapy compared to $32.8 \%$ MSS stage B cancers $(P=0.2)$, and $68.4 \%$ of MSI-H stage $\mathrm{C}$ cancers received adjuvant therapy compared to $75.3 \%$ of MSS stage $C$ cancers $(P=0.4)$. Adjuvant therapy did not significantly affect survival for localized or metastatic disease (data not shown). 
Table 1 Association of clinicopathologic features with MSI in mucinous colorectal cancer

\begin{tabular}{|c|c|c|c|}
\hline & $M S I(\mathrm{n}=72)$ & $M S S(\mathrm{n}=176)$ & P-value \\
\hline \multicolumn{4}{|l|}{ Gender } \\
\hline Female & $40(55.6)$ & $83(47.2)$ & \multirow[t]{2}{*}{0.27} \\
\hline Male & $32(44.4)$ & $93(52.8)$ & \\
\hline \multicolumn{4}{|l|}{ Age (years) } \\
\hline$<70$ & $29(40.3)$ & $89(50.6)$ & \multirow[t]{2}{*}{0.061} \\
\hline$>70$ & $43(59.7)$ & $87(49.4)$ & \\
\hline \multicolumn{4}{|l|}{ Site $^{\mathrm{a}}$} \\
\hline Right & $60(83.3)$ & 85 (48.9) & \multirow[t]{2}{*}{$<0.001$} \\
\hline Left & $12(16.7)$ & $90(51.1)$ & \\
\hline \multicolumn{4}{|l|}{ Tumor size ${ }^{\mathrm{a}}$} \\
\hline$<6 \mathrm{~cm}$ & $38(52.8)$ & $92(52.3)$ & \multirow[t]{2}{*}{0.89} \\
\hline$\geq 6 \mathrm{~cm}$ & $34(47.2)$ & $82(47.7)$ & \\
\hline \multicolumn{4}{|l|}{ Margins } \\
\hline Pushing & $30(41.7)$ & $55(31.2)$ & \multirow[t]{2}{*}{0.10} \\
\hline Infiltrating & $42(58.3)$ & $121(68.8)$ & \\
\hline \multicolumn{4}{|l|}{ Grade } \\
\hline 1 & 0 & $1(0.5)$ & \multirow{4}{*}{0.21} \\
\hline 2 & $34(47.9)$ & 105 (61.3) & \\
\hline 3 & $31(43.7)$ & $58(33.6)$ & \\
\hline 4 & $6(8.4)$ & $8(4.6)$ & \\
\hline \multicolumn{4}{|l|}{ Pattern } \\
\hline Pure mucinous & $48(66.7)$ & $132(75.0)$ & \multirow[t]{2}{*}{0.18} \\
\hline Mixed & $24(33.3)$ & $44(25.0)$ & \\
\hline \multicolumn{4}{|l|}{ Vascular invasion } \\
\hline Absent & $58(80.6)$ & $142(80.7)$ & \multirow[t]{2}{*}{0.86} \\
\hline Present & $14(19.4)$ & $34(19.3)$ & \\
\hline \multicolumn{4}{|c|}{ Crohn's-like infiltrate } \\
\hline Absent & $24(33.3)$ & $122(69.3)$ & \multirow[t]{2}{*}{$<0.001$} \\
\hline Present & $48(66.7)$ & $54(30.7)$ & \\
\hline \multicolumn{4}{|l|}{ TIL } \\
\hline$<2$ per $10 \mathrm{HPF}$ & $18(25.0)$ & 139 (79.0) & \multirow[t]{2}{*}{$<0.001$} \\
\hline$>2$ per $10 \mathrm{HPF}$ & $54(75.0)$ & $37(21.0)$ & \\
\hline \multicolumn{4}{|l|}{ Stage } \\
\hline A & $2(2.8)$ & $1(0.6)$ & \multirow[t]{4}{*}{$<0.001$} \\
\hline $\mathrm{B}$ & $48(66.7)$ & $67(38.1)$ & \\
\hline $\mathrm{C}$ & $19(26.4)$ & $79(44.9)$ & \\
\hline $\mathrm{D}$ & $3(4.1)$ & $29(16.5)$ & \\
\hline
\end{tabular}

${ }^{\mathrm{a}}$ Site unknown in one case; size unknown in two cases; grade unknown in five cases.

Univariate analysis identified low stage, low grade, right-sided location, absence of vascular invasion, high TIL count, presence of Crohn's-like reaction and MSI-H status as having favorable influence on age-independent survival (Table 2 and Figure 1). The survival benefit of MSI-H status was not independent of stage. In multivariate analysis, older age, higher grade, higher stage and absence of Crohn's-like infiltrate were independent predictors of poor outcome (Table 3). Neither TIL count nor MSI status were significant prognostic factors in multivariate analysis.

\section{Discussion}

We found that about one-third of mucinous carcinomas $(29.3 \%)$ are MSI-H. The reported prevalence for all colon cancers is $15-20 \%$. Our result could be a slight underestimation, because we used immunohistochemical staining for mismatch repair enzymes
Table 2 Impact of factors on survival in colorectal mucinous carcinoma, adjusted for age at surgery, as estimated by the Cox model

\begin{tabular}{lccr}
\hline Variable & $\begin{array}{c}\text { Hazard } \\
\text { ratio }\end{array}$ & $\begin{array}{c}\text { 95\% Confidence } \\
\text { limits }\end{array}$ & P-value \\
\hline Male sex & 0.926 & $0.654-1.312$ & 0.6669 \\
Right sided & 0.635 & $0.443-0.911$ & 0.0137 \\
location & & & \\
Tumor size & 1.013 & $0.958-1.072$ & 0.6446 \\
Margins & 0.749 & $0.509-1.101$ & 0.1410 \\
Vascular invasion & 1.668 & $1.044-2.665$ & 0.0323 \\
Grade & 1.456 & $1.118-1.897$ & 0.0053 \\
Crohn's-like & 0.588 & $0.404-0.856$ & 0.0056 \\
infiltrate & & & \\
TIL >2/10 HPF & 0.650 & $0.444-0.951$ & 0.0263 \\
MSI & 0.597 & $0.390-0.914$ & 0.0176 \\
Stage & 2.849 & $2.168-3.742$ & $<0.0001$ \\
\hline
\end{tabular}

$\mathrm{TIL}=$ tumor-infiltrating lymphocytes; HPF = high-power fields.

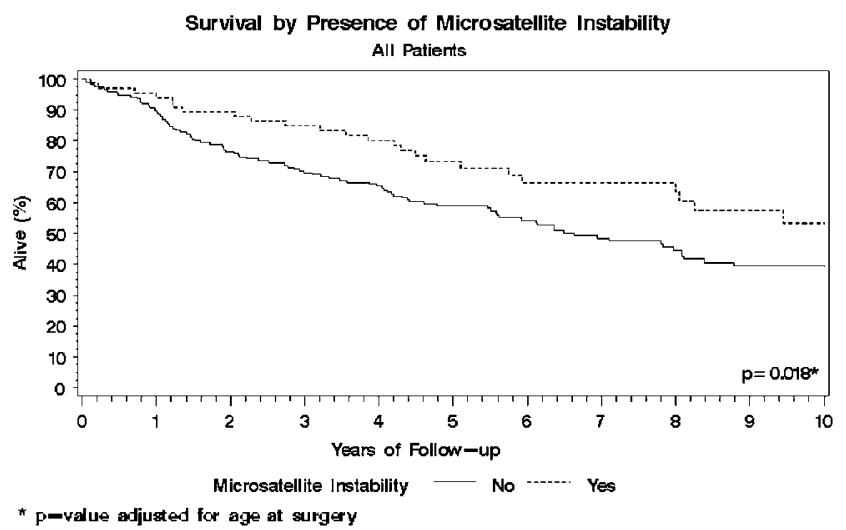

Figure 1 Kaplan-Meier curves demonstrating survival by microsatellite instability status: all patients.

Table 3 Multivariate Cox proportional hazards model predicting death in mucinous colorectal cancer

\begin{tabular}{lccr}
\hline Variable & $\begin{array}{c}\text { Hazard } \\
\text { ratio }\end{array}$ & $\begin{array}{c}\text { 95\% hazard } \\
\text { ratio confidence } \\
\text { limits }\end{array}$ & P-value \\
\hline Age & 1.039 & $1.019-1.059$ & $<0.0001$ \\
MSI & 1.008 & $0.561-1.808$ & 0.9798 \\
Stage & 3.155 & $2.193-4.538$ & $<0.0001$ \\
Grade & 1.378 & $1.034-1.837$ & 0.0285 \\
$\begin{array}{l}\text { Crohn's-like } \\
\text { infiltrate }\end{array}$ & 0.634 & $0.426-0.944$ & 0.0248 \\
\hline
\end{tabular}

as a marker for MSI. The correlation between immunohistochemistry and microsatellite analysis by PCR is essentially perfect for MSI caused by methylation of the hMLH1 promotor region, but occasional hereditary mutations eliminate mismatch repair function while retaining stainable protein. ${ }^{28-30}$ HNPCC is estimated to account for $1-2 \%$ of colorectal cancer. $^{2}$ Our three patients with loss of 
hMSH2 staining almost certainly carried germline mutations, ${ }^{4,31}$ so our yield of 3/248 makes it unlikely that our approach missed many hereditary cancers. It is also conceivable that derangement of other mismatch repair proteins such as PMS2 resulted in microsatellite instability not detected by our stains, but those too are rare events. ${ }^{32}$

The clinicopathologic profile of MSI-H mucinous carcinoma is similar to that of MSI-H carcinoma in general: right-sided location, low stage at presentation, many TILs and a prominent Crohn's-like infiltrate. We found no correlation between MSI status and growth pattern in mucinous carcinoma. Our findings differ from those of those of the nextlargest study of mucinous carcinoma; Messerini et $a l^{33}$ reviewed 50 mucinous carcinomas and found a positive correlation between MSI-H and expanding growth pattern, but no correlation with tumor site, lymphocytes or Crohn's-like infiltrate.

Our patients with MSI-H mucinous carcinoma had significantly better age-adjusted survival than did those with MSS mucinous cancer $(P=$ 0.0176). Comparisons within stage, however, failed to show differences in survival for same-stage tumors. Multivariate analysis bore out this observation, demonstrating that MSS was not an independent predictor of prognosis. Instead, patient age, tumor grade, tumor stage and Crohn's-like infiltrate proved to be independent variables affecting outcome. MSI-H mucinous carcinoma was much less likely than MSS mucinous carcinoma to present with lymph node metastases or distant metastases $(P<0.001)$, accounting for its favorable prognosis overall.

The relative inability of MSI-H tumors to develop nodal or distant metastases has been noted in several studies. ${ }^{9,34}$ It has been hypothesized that the absence of chromosomal instability and a lower incidence of p53 mutations in MSI-H tumors may be responsible for the delayed progression to metastatic disease. ${ }^{15}$ For our patients with nodal or distant metastases at presentation, the prognosis for MSI-H mucinous carcinoma was not different from microsatellite stable mucinous carcinoma. This is in contrast to several large studies that have shown a survival benefit for patients with lymph nodepositive MSI-H cancers, but those studies did not divide the tumors based on histologic type, and can say nothing about mucinous carcinoma in particular. ${ }^{12-14}$ It has also been suggested that adjuvant chemotherapy confers a strongly favorable effect on outcome in node-positive MSI-H colorectal cancer. ${ }^{13,14}$ Our patients were equally likely to have received adjuvant therapy regardless of microsatellite instability status, and therapy status did not appear to influence survival for either MSI-H or microsatellite stable cancers. It may be that mucinous carcinoma is biologically distinct from conventional adenocarcinoma in terms of response to adjuvant therapy, but our retrospective study cannot answer that question.
A prominent host lymphoid response has been a consistent finding in MSI-H carcinoma,,$^{5-8,35,36}$ and our study documented an association between MSI$\mathrm{H}$ mucinous carcinoma and both the Crohn's-like infiltrate and increased numbers of TILs. Both features have been correlated with favorable outcome. ${ }^{37-42}$ In our series, high numbers of TILs were associated with better survival in univariate but not in multivariate analysis. The Crohn's-like reaction, in contrast, did retain statistical significance in multivariate analysis as a marker of favorable prognosis.

MSI-H mucinous carcinomas are right-sided, lowstage tumors with Crohn's-like reaction and TILs. The prognosis for MSI-H mucinous carcinoma is better than for microsatellite stable mucinous carcinoma, but the difference is related to lower stage at presentation and prominent Crohn's-like infiltrate. These two parameters can be evaluated with routine pathologic techniques. While the assessment of MSI status may add value in the setting of suspected HNPCC, it is not necessary to determine MSI status solely to stratify outcome for mucinous carcinoma.

\section{References}

1 Peltomaki P, Vasen HF. Mutations predisposing to hereditary nonpolyposis colorectal cancer: database and results of a collaborative study. The International Collaborative Group on Hereditary Nonpolyposis Colorectal Cancer. Gastroenterology 1997;113:1146-1158.

2 Aaltonen LA, Salovaara R, Kristo P, et al. Incidence of hereditary nonpolyposis colorectal cancer and the feasibility of molecular screening for the disease. N Engl J Med 1998;338:1481-1487.

3 Thibodeau SN, Bren G, Schaid D. Microsatellite instability in cancer of the proximal colon. Science 1993;260:816-819.

4 Thibodeau SN, French AJ, Cunningham JM, et al. Microsatellite instability in colorectal cancer: different mutator phenotypes and the principal involvement of hMLH1. Cancer Res 1998;58:1713-1718.

$5 \mathrm{Kim} \mathrm{H}$, Jen J, Vogelstein B, et al. Clinical and pathological characteristics of sporadic colorectal carcinomas with DNA replication errors in microsatellite sequences. Am J Pathol 1994;145:148-156.

6 Risio M, Reato G, di Celle PF, et al. Microsatellite instability is associated with the histological features of the tumor in nonfamilial colorectal cancer. Cancer Res 1996;56:5470-5474.

7 Jass JR, Do KA, Simms LA, et al. Morphology of sporadic colorectal cancer with DNA replication errors. Gut 1998;42:673-679.

8 Smyrk TC, Watson P, Kaul K, et al. Tumor-infiltrating lymphocytes are a marker for microsatellite instability in colorectal cancer. Cancer 2001;91:2417-2422.

9 Jernvall P, Makinen MJ, Karttunen TJ, et al. Microsatellite instability: impact on cancer progression in proximal and distal colorectal cancers. Eur J Cancer 1999;35:197-201.

10 Liang JT, Chang KJ, Chen JC, et al. Clinicopathologic and carcinogenetic appraisal of DNA replication error in sporadic T3N0M0 stage colorectal cancer after 
curative resection. Hepatogastroenterol 1999;46: 883-890.

11 Gryfe R, Kim H, Hsieh ET, et al. Tumor microsatellite instability and clinical outcome in young patients with colorectal cancer. N Engl J Med 2000;342:69-77.

12 Wright CM, Dent OF, Barker M, et al. Prognostic significance of extensive microsatellite instability in sporadic clinicopathological stage C colorectal cancer. Br J Surg 2000;87:1197-1202.

13 Hemminki A, Mecklin JP, Jarvinen H, et al. Microsatellite instability is a favorable prognostic indicator in patients with colorectal cancer receiving chemotherapy. Gastroenterology 2000;119:921-928.

14 Elsaleh H, Joseph D, Grieu F, et al. Association of tumour site and sex with survival benefit from adjuvant chemotherapy in colorectal cancer. Lancet 2000;355:1745-1750.

15 Elsaleh H, Powell B, McCaul K, et al. p53 alteration and microsatellite instability have predictive value for survival benefit from chemotherapy in stage III colorectal carcinoma. Clin Cancer Res 2001;7:1343-1349.

16 Samowitz WS, Curtin K, Ma KN, et al. Microsatellite instability in sporadic colon cancer is associated with an improved prognosis at the population level. Cancer Epidemiol Biomarkers Prev 2001;10:917-923.

17 Green JB, Timmcke AE, Mitchell WT, et al. Mucinous carcinoma-just another colon cancer? Dis Colon Rectum 1993;36:49-54.

18 Minsky BD, Mies C, Rich TA, et al. Colloid carcinoma of the colon and rectum. Cancer 1987;60: 3103-3112.

19 Secco GB, Fardelli R, Campora E, et al. Primary mucinous adenocarcinomas and signet-ring cell carcinomas of colon and rectum. Oncology 1994;51:30-34.

20 Consorti F, Lorenzotti A, Midiri G, et al. Prognostic significance of mucinous carcinoma of colon and rectum: a prospective case-control study. J Surg Oncol 2000;73:70-74.

21 Symonds DA, Vickery AL. Mucinous carcinoma of the colon and rectum. Cancer 1976;37:1891-1900.

22 Suma KS, Nirmala V. Mucinous component in colorectal carcinoma-prognostic significance: a study in a south Indian population. J Surg Oncol 1992;51:60-64.

23 Connelly JH, Robey-Cafferty SS, Cleary KR. Mucinous carcinomas of the colon and rectum. An analysis of 62 stage B and C lesions. Arch Pathol Lab Med 1991;115: 1022-1025.

24 Enriquez JM, Diez M, Tobaruela E, et al. Clinical, histopathological, cytogenetic and prognostic differences between mucinous and nonmucinous colorectal adenocarcinomas. Rev Esp Enferm Dig 1998;90: $563-572$.

25 Messerini L, Ciantelli M, Baglioni S, et al. Prognostic significance of microsatellite instability in sporadic mucinous colorectal cancers. Hum Pathol 1999;30: 629-634.

26 Compton C, Fenoglio-Preiser CM, Pettigrew N, et al. American Joint Committee on Cancer Prognostic
Factors Consensus Conference: Colorectal Working Group. Cancer 2000;88:1739-1757.

27 Compton CC, Fielding LP, Burgart LJ, et al. Prognostic factors in colorectal cancer. College of American Pathologists Consensus Statement 1999. Arch Pathol Lab Med 2000;124:979-994.

28 Marcus VA, Madlensky L, Gryfe R, et al. Immunohistochemistry for hMLH1 and hMSH2: a practical test for DNA mismatch repair-deficient tumors. Am J Surg Pathol 1999;23:1248-1255.

29 Perrin J, Gouvernet J, Parriaux D, et al. MSH2 and MLH1 immunodetection and the prognosis of colon cancer. Int J Oncol 2001;19:891-895.

30 Lindor NM, Burgart LJ, Leontovich O, et al. Immunohistochemistry versus microsatellite instability testing in phenotyping colorectal tumors. J Clin Oncol 2002; 20:1043-1048.

31 Herman JG, Umar A, Polyak K, et al. Incidence and functional consequences of hMLH1 promoter hypermethylation in colorectal carcinoma. Proc Natl Acad Sci USA 1998;95:6870-6875.

32 De Rosa M, Fasano C, Panariello L, et al. Evidence for a recessive inheritance of Turcot's syndrome caused by compound heterozygous mutations within the PMS2 gene. Oncogene 2000;23:1719-1723.

33 Messerini L, Vitelli F, De Vitis LR, et al. Microsatellite instability in sporadic mucinous colorectal carcinomas: relationship to clinico-pathological variables. J Pathol 1997;182:380-384.

34 Jass JR, Smyrk, TC, Stewart SM, et al. Pathology of hereditary non-polyposis colorectal cancer. Anticancer Res 1994;14:1631-1634.

35 Ward R, Meagher A, Tomlinson I, et al. Microsatellite instability and the clinicopathological features of sporadic colorectal cancer. Gut 2001;48:821-829.

36 Alexander J, Watanabe T, Wu TT, et al. Histopathological identification of colon cancer with microsatellite instability. Am J Pathol 2001;158:527-535.

37 Graham DM, Appelman HD. Crohn's-like lymphoid reaction and colorectal carcinoma: a potential histologic prognosticator. Mod Pathol 1990;3:332-335.

38 Harrison JC, Dean PJ, el-Zeky F, et al. Impact of the Crohn's-like lymphoid reaction on staging of rightsided colon cancer: results of multivariate analysis. Hum Pathol 1995;26:31-38.

39 Diez M, Pollan M, Enriquez JM, et al. Histopathologic prognostic score in colorectal adenocarcinomas. Anticancer Res 1998;18:689-694.

40 Watt AG, House AK. Colonic carcinoma. A quantitative assessment of lymphocyte infiltration of the periphery of colonic tumors related to prognosis. Cancer 1978;41:279-282.

41 Jass JR. Lymphocytic infiltration and survival in rectal cancer. J Clin Pathol 1986;39:585-589.

42 Di Giorgio A, Botti C, Tocchi A, et al. The influence of tumor lymphocytic infiltration on long term survival of surgically treated colorectal cancer patients. Int Surg 1992;77:256-260. 